\title{
Degradación ambiental de las propiedades mecánicas de fibras monocristallinas de circona dopada con erbia
}

\author{
J. Y. PASTOR ${ }^{1}$, Á. RIDRUEJO ${ }^{1}$, J. LLORCA ${ }^{1}$, A. SAYIR ${ }^{2}$, VÍCTOR M. ORERA ${ }^{3}$ \\ 'Departamento de Ciencia de Materiales. Universidad Politécnica de Madrid. \\ E. T. S. de Ingenieros de Caminos, Canales y Puertos. 28040 Madrid. \\ ${ }^{2}$ NASA Glenn Research Center, Cleveland, OH 44135-3191, U. S. A. \\ ${ }^{3}$ Instituto de Ciencia de Materiales de Aragón \\ C.S.I.C.- Universidad de Zaragoza. 50009 - Zaragoza.
}

\begin{abstract}
Partiendo de fibras monocristalinas de $\mathrm{ZrO}_{2}\left(\mathrm{Er}_{2} \mathrm{O}_{3}\right)$ tetragonal fabricadas por un procedimiento de fusión zonal con láser (LHFZ), se efectuaron ensayos de flexión en los que se medía la resistencia mecánica en función de la temperatura (25 a $800^{\circ} \mathrm{C}$ ) y el medio (aire o agua). Los ensayos fueron realizados a distintas velocidades con objeto de establecer la susceptibilidad a las condiciones ambientales. La susceptibilidad ambiental de la $\mathrm{ZrO}\left(\mathrm{Y}_{2} \mathrm{O}_{3}\right)$ se había asociado siempre a la desestabilización de la fase tetragonal, pero el análisis de las superficies de fractura mediante microscopía electrónica de barrido y espectroscopia Raman confocal muestran una ausencia completa de fase monoclínica en las superficies de fractura de las fibras. Esta ausencia, junto con los ensayos complementarios sobre fibras sometidas a tratamientos de recocido para eliminar las tensiones residuales provenientes del procesado, indican que el crecimiento subcrítico de grieta se debe a un mecanismo de corrosión bajo tensión.
\end{abstract}

Palabras clave: circona, erbia, estructura tetragonal, susceptibilidad al ambiente, crecimiento subcrítico de grieta, corrosión bajo tensión.

Environmental degradation of the mechanical properties of single-crystal erbia-doped zircona fibers

The flexure strength of tetragonal $\mathrm{ZrO}_{2}\left(\mathrm{Er}_{2} \mathrm{O}_{3}\right)$ single-crystal monofilaments manufactured by the laser-heated floating zone method was measured as a function of the environment (air vs. water) and temperature (from 25 to $800^{\circ} \mathrm{C}$ ) at loading rates spanning three orders of magnitude to ascertain their susceptibility to the environmental conditions. These mechanical tests were completed with parallel tests on fully-annealed monofilaments (to relieve the thermal residual stresses induced during growth) and by detailed analysis of the fracture surfaces using scanning electron microscopy and micro-Raman spectroscopy. While environmental susceptibility of $\mathrm{ZrO}_{2}\left(\mathrm{Y}_{2} \mathrm{O}_{3}\right)$ in previous investigations was always associated with the destabilization of the tetragonal phase, monoclinic phase was not detected on the fracture surfaces of the $\mathrm{ZrO}_{2}\left(\operatorname{Er}_{2} \mathrm{O}_{3}\right)$ monofilaments and it was concluded that slow crack growth in this material at high temperature or immersed in water was due to stress-corrosion cracking.

Keywords: zircona, erbia, tetragonal structure, environmental susceptibility, slow crack growth, stress-corrosion cracking

\section{INTRODUCCIÓN}

La importancia de la circona como material estructural estriba principalmente en la capacidad de aumentar su tenacidad mediante la transformación de la fase tetragonal -metaestable- en fase monoclínica cuando en el entorno de la punta de la grieta se supera un determinado umbral de tensión. La literatura científica [1-3] ha descrito detalladamente algunos de los factores relevantes, tales como la cantidad y naturaleza de los elementos estabilizantes, el tamaño de grano o la presencia de tensiones residuales. Sin embargo, una de las limitaciones que presentan los materiales cerámicos de circona, de cara a su aplicación industrial es la degradación ambiental. Se ha observado dicha degradación en $\mathrm{ZrO}_{2}\left(\mathrm{Y}_{2} \mathrm{O}_{3}\right)$ tras largos períodos de permanencia a temperaturas entre 200 y $300^{\circ} \mathrm{C}$ [8-9], o en entornos húmedos [10-11].

Dentro de este contexto, la circona dopada con erbia es un material prometedor. Aparte del gran poder estabilizador de la fase tetragonal que posee la erbia, las fuertes interacciones entre dislocaciones y los iones $\mathrm{Er}^{3+}$ hacen que la resistencia a fluencia de monocristales de $\mathrm{ZrO}_{2}\left(\mathrm{Er}_{2} \mathrm{O}_{3}\right)$ sean mayores que las de $\mathrm{ZrO}_{2}\left(\mathrm{Y}_{2} \mathrm{O}_{3}\right)$ [7]. No obstante, dadas las similaridades entre circona dopada con erbia y la variante dopada con itria, es necesario averiguar si la degradación de $\mathrm{ZrO}_{2}\left(\mathrm{Y}_{2} \mathrm{O}_{3}\right)$ aparece también en $\mathrm{ZrO}_{2}\left(\mathrm{Er}_{2} \mathrm{O}_{3}\right)$. Para ello, se utilizó la fusión zonal por láser para fabricar filamentos monocristalinos de $\mathrm{ZrO}_{2}\left(\mathrm{Er}_{2} \mathrm{O}_{3}\right)$. Posteriormente, estos monofilamentos se ensayaron a flexión en tres puntos entre 25 y $800{ }^{\circ} \mathrm{C}$, así como en medio acuoso. El programa experimental se completó con ensayos sobre fibras recocidas (para eliminar las tensiones residuales experimentadas durante el procesado) y con un análisis detallado de las superficies de fractura mediante microscopía electrónica de barrido y espectroscopia micro-Raman, que permiten estudiar los posibles factores de deterioro del material. 


\section{TÉCNICAS EXPERIMENTALES}

Los monofilamentos, con un contenido en $\mathrm{Er}_{2} \mathrm{O}_{3}$ del $5 \%$ molar, se prepararon a partir de barras extruidas de material precursor (pasta Alpha Aesar, 99.999\% de pureza). Los detalles acerca del procedimiento de fusión zonal por láser se describen en las referencias [12-13]. El monocristal se hizo crecer con orientación $<111>$ gracias a una semilla de $\mathrm{ZrO}_{2}\left(\mathrm{Y}_{2} \mathrm{O}_{3}\right)$ a una velocidad de $38 \mathrm{~cm} / \mathrm{h}$ hasta alcanzar una longitud de $20 \mathrm{~cm}$. El diámetro medio estaba en torno a $170 \mu \mathrm{m}$, si bien existían variaciones significativas tanto entre distintos monofilamentos (130 a $230 \mu \mathrm{m}$ ) como a lo largo de cada uno de ellos (ver Fig. 1). La microestructura se compone de tres variantes cristalinas tetragonales interpenetradas con los ejes c perpendiculares y el eje $<111>$ siempre paralelo a la dirección de crecimiento [7].

$500 \mu \mathrm{m}$

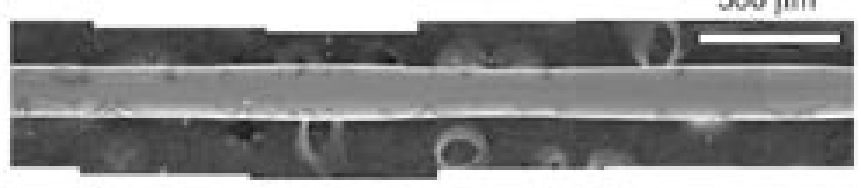

Fig. 1- Tramo de fibra monocristalina de $\mathrm{ZrO}_{2}\left(\mathrm{Er}_{2} \mathrm{O}_{3}\right)(5 \%$ molar $)$ tetragonal fabricado mediante fusión zonal por láser

Para medir la resistencia a flexión, se mecanizó en alúmina un dispositivo para ensayo en tres puntos, con una distancia entre apoyos de $1.5 \mathrm{~mm}$. El dispositivo se conectó al actuador de una máquina universal (Instron 8501) y se introdujo dentro de un horno modificado (MTS, modelo 653) para permitir ensayar a alta temperatura. El alineamiento de todos los componentes se conseguía mediante una mesa de coordenadas acoplada al actuador. Para asegurar la perpendicularidad del monofilamento respecto de los rodillos de apoyo se contaba además con la ayuda de una lupa binocular. Se programó una velocidad de calentamiento de $20^{\circ} \mathrm{C} / \mathrm{min}$ y un tiempo de permanencia a la temperatura de ensayo de 20 minutos para permitir acomodar las dilataciones y alcanzar la estabilidad completa del sistema. La carga se medía con una célula de 10 $\mathrm{N}$, y la flecha con un LVDT. Se efectuaron ensayos controlando el desplazamiento en atmósfera de laboratorio a velocidades entre 1 y $100 \mu \mathrm{m} / \mathrm{min}$ a $25^{\circ} \mathrm{C}$ y $800{ }^{\circ} \mathrm{C}$, mientras que a 600 ${ }^{\circ} \mathrm{C}$ fueron realizados entre 1 y $10 \mu \mathrm{m} / \mathrm{min}$; y aquellos a 400 ${ }^{\circ} \mathrm{C}$, únicamente a $10 \mu \mathrm{m} / \mathrm{min}$. Las curvas carga-flecha eran siempre lineales hasta la rotura, y se utilizó el modelo de Euler-Bernouilli para vigas de sección circular para calcular la tensión de rotura. El diámetro de la sección de rotura se había medido previamente mediante un microscopio óptico, y después de la rotura sobre la superficie de yactura en un MEB siempre que fue posible (la pequeña masa de la probeta y la elevada cantidad de energía elástica almacenada hace que las probetas salgan despedidas y se rompan en varios trozos o sea imposible encontrarlas).

A altas temperaturas, en numerosas ocasiones la probeta se rompía en varias piezas, con lo que no siempre era posible identificar las superficies correspondientes a la fractura inicial. Las que sí pudieron identificarse se examinaron con el microscopio electrónico (JEOL 6300) para examinar el origen y características micromorfológicas de la fractura. Con objeto de determinar la presencia de $\mathrm{ZrO}_{2}$ monoclínica después de los ensayos, se efectuaron también diversas medidas por espectroscopia Raman en las superficies de fractura y laterales de las fibras rotas. El equipo contaba con un espectrómetro con detector multicanal (Dilor XY) y se utilizaba un microscopio óptico con apertura de $400 \mu \mathrm{m}$ en el plano focal imagen para analizar un área de $1 \mu \mathrm{m}$ de diámetro y $4 \mu \mathrm{m}$ de profundidad. Para evitar que la intensa línea de emisión del nivel ${ }^{4} S_{3 / 2}$ del ión $\mathrm{Er}^{3+}$ enmascarara la señal, se utilizaron para las medidas o bien la longitud de onda $457.9 \mathrm{~nm}$ del láser de argón (Innova 200), o bien la de $575.6 \mathrm{~nm}$ del láser de colorante.

\section{RESULTADOS}

\subsection{Efecto de la temperatura}

Los ensayos de flexión en tres puntos se realizaron a temperaturas de 25,600 y $800{ }^{\circ} \mathrm{C}$ a distintas velocidades de deformación $1 \mu \mathrm{m} / \mathrm{min}, 10 \mu \mathrm{m} / \mathrm{min}$ y $100 \mu \mathrm{m} / \mathrm{min}$. Se efectuaron entre 4 y 8 ensayos de cada tipo dependiendo de la dispersión encontrada. En las figuras 2, 3 y 4 se muestra la tensión de rotura en función de la velocidad

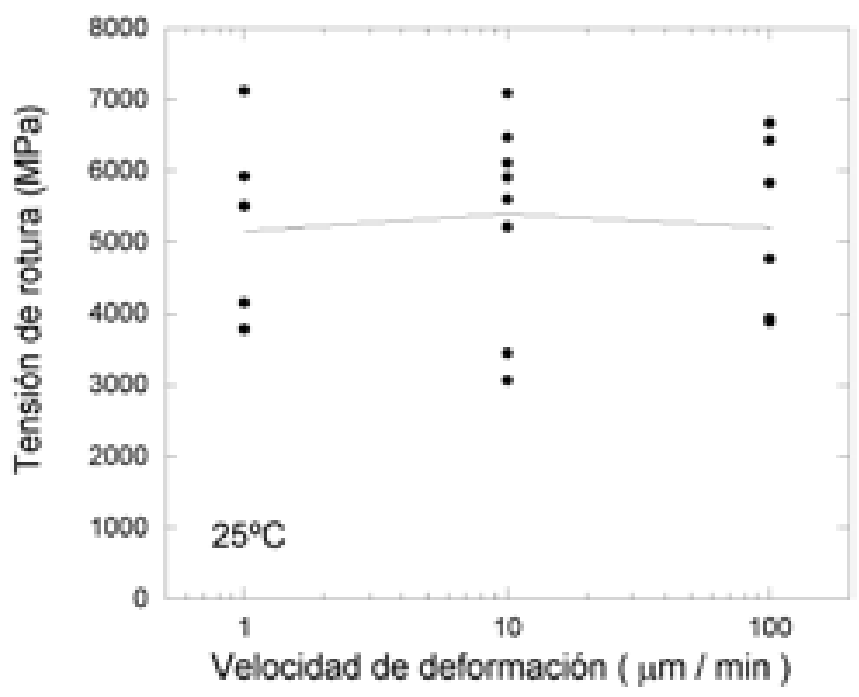

Fig. 2- Resistencia a flexión de los monofilamentos de $\mathrm{ZrO}_{2}\left(\mathrm{Er}_{2} \mathrm{O}_{3}\right)$ a 25 ${ }^{\circ} \mathrm{C}$ en función de la velocidad de deformación.

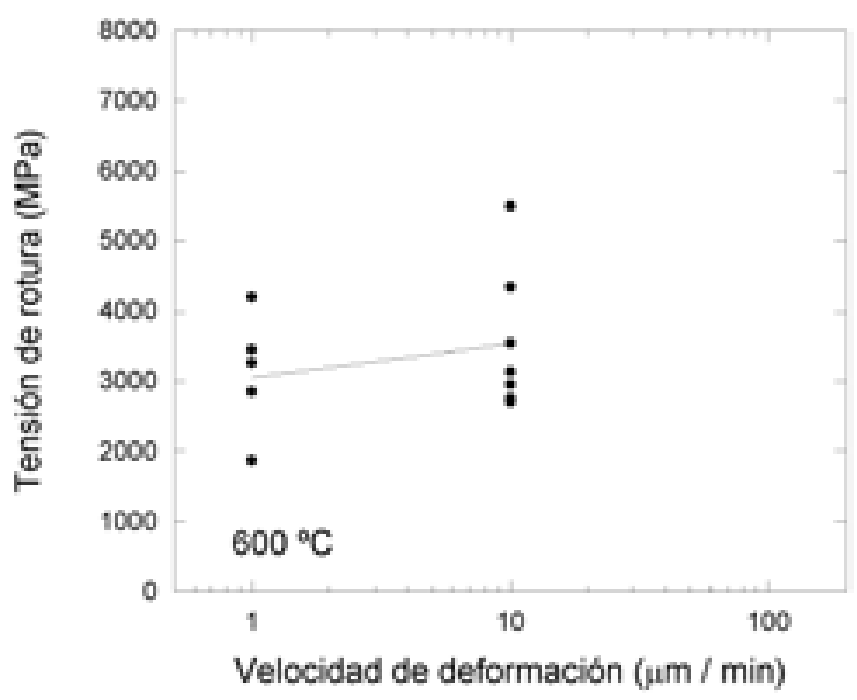

Fig. 3- Resistencia a flexión de los monofilamentos de $\mathrm{ZrO}_{2}\left(\mathrm{Er}_{2} \mathrm{O}_{3}\right)$ a $600{ }^{\circ} \mathrm{C}$ en función de la velocidad de deformación. 


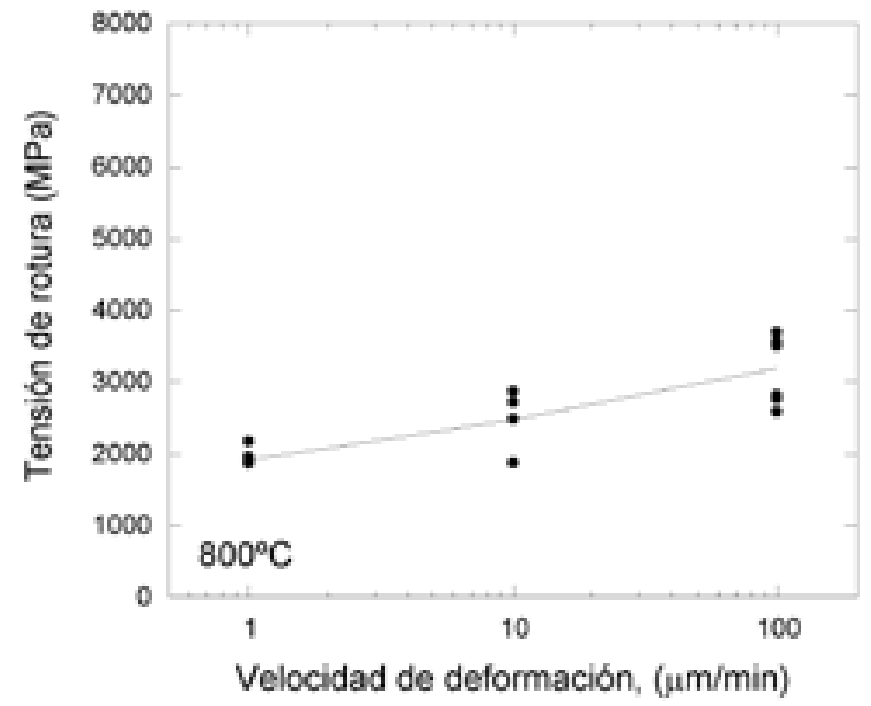

Fig. 4- Resistencia a flexión de los monofilamentos de $\mathrm{ZrO}_{2}\left(\mathrm{Er}_{2} \mathrm{O}_{3}\right)$ a $800{ }^{\circ} \mathrm{C}$ en función de la velocidad de deformación.

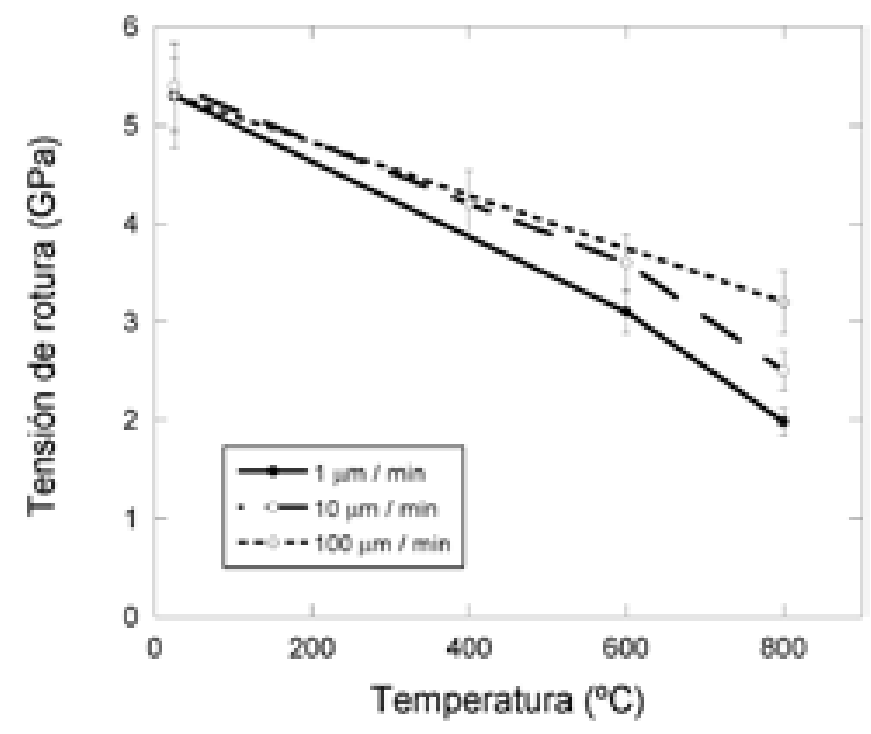

Fig. 5- Resistencia media a flexión de los monofilamentos según la temperatura y la velocidad de deformación.

de deformación. Como puede observarse, a temperatura ambiente la resistencia de las fibras no depende de la velocidad de deformación, mientras que a $600{ }^{\circ} \mathrm{C}$, y más marcadamente a $800{ }^{\circ} \mathrm{C}$, se comprueba cómo la resistencia se reduce a medida que disminuye la velocidad de ensayo. La figura 5, que incluye datos tomados a $400{ }^{\circ} \mathrm{C}$, resume la dependencia de la tensión de rotura con la temperatura y muestra un decaimiento sostenido de la resistencia. Además, en el análisis de los resultados individuales de cada uno de los ensayos (no recogidos en la gráfica) se apreció cómo la gran dispersión de tensiones de rotura que existe a $25{ }^{\circ} \mathrm{C}$ (valores mínimos de 3-4 GPa y máximos de $\sim 7 \mathrm{GPa}$ ) se reduce en los ensayos a alta temperatura, llegando a un mínimo a $800{ }^{\circ} \mathrm{C}$. Esto indica que los mecanismos responsables de la fractura no eran los mismos a temperatura ambiente y a alta temperatura.

Además de los datos proporcionados por los meros ensayos mecánicos, los análisis de las superficies de rotura
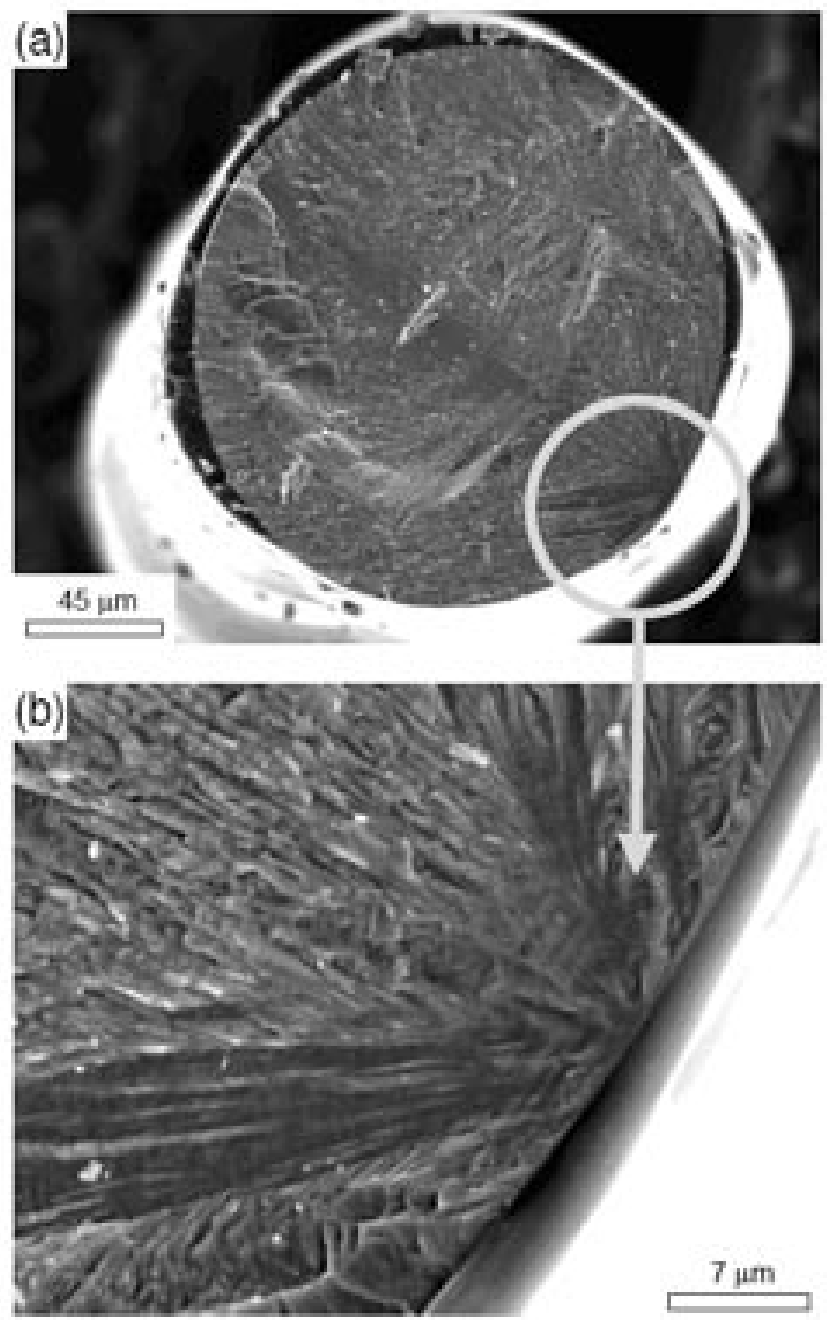

Fig. 6- Superficie de fractura de muestra ensayada a $25^{\circ} \mathrm{C}$. (a) Vista general. (b) Detalle de la zona de inicio de la fractura.

revelaron cambios morfológicos. Los monofilamentos ensayados a $25{ }^{\circ} \mathrm{C}$ mostraban superficies rugosas (Figura $6 a)$, con surcos apuntando hacia el origen de la fractura, que resultaba ser siempre algún pequeño defecto en la superficie de la fibra (Fig. 6b). El tamaño y la posición de estos defectos superficiales condicionan la tensión de rotura, y de ahí la gran dispersión que presentan, típica de los materiales cerámicos frágiles. Sin embargo, ya a $600{ }^{\circ} \mathrm{C}$, las superficies de rotura son claramente más lisas (Fig. 7). Los surcos aparecen irradiados desde una zona extensa tipo espejo (mirror), compatible con una situación de crecimiento lento del frente de grieta (crecimiento subcrítico de grieta) hasta alcanzar el tamaño crítico a partir del cual se produce la rotura frágil (Fig.7). Este mecanismo concordaría con la dependencia acusada de la resistencia a flexión con la velocidad de deformación y con la reducción en su dispersión a 600 y $800{ }^{\circ} \mathrm{C}$, puesto que sería ahora la velocidad de crecimiento de grieta (que depende de la temperatura de ensayo), y no el tamaño del defecto inicial, la que determinaría la resistencia mecánica de las muestras.

\subsection{Efecto del ambiente}

Las muestras se sumergieron en agua durante 24 horas antes de ser ensayadas mecánicamente para comprobar su 

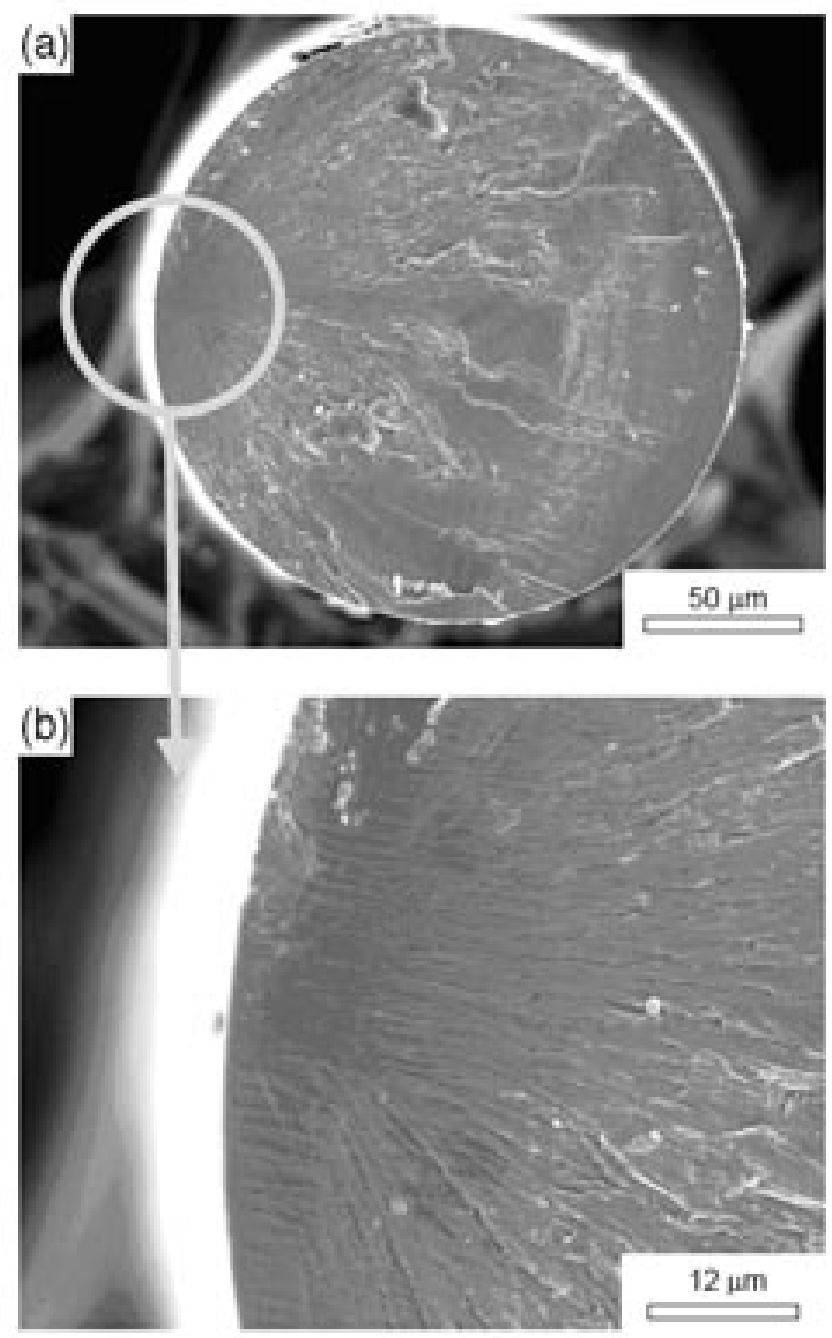

Fig. 7- Superficie de fractura de muestra ensayada a $600{ }^{\circ} \mathrm{C}$. (a) Vista general. (b) Detalle de la zona de inicio de la fractura.

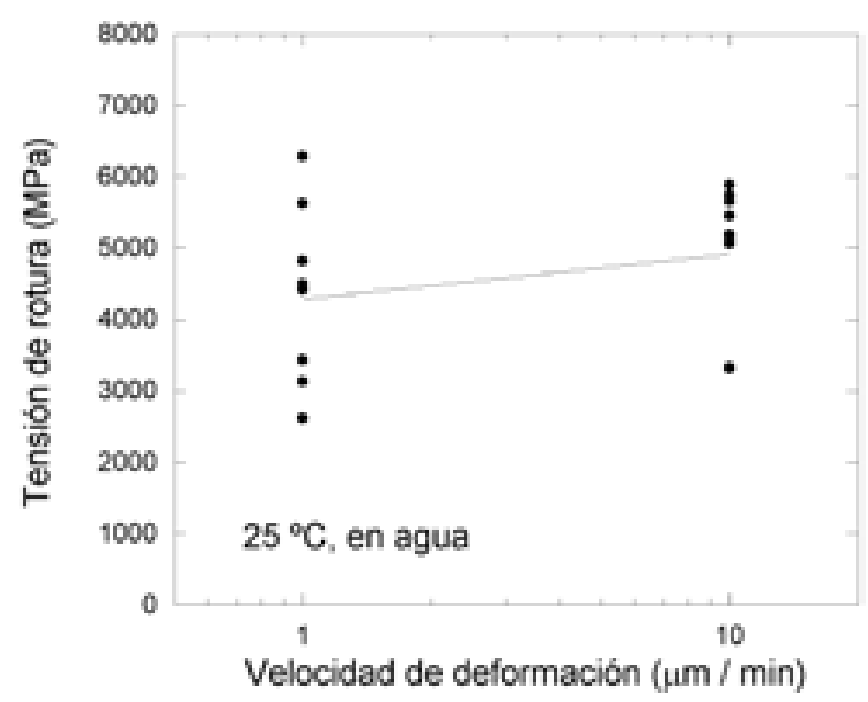

Fig. 8- Resistencia media a flexión de los monofilamentos de $\mathrm{ZrO}_{2}$ $\left(\mathrm{Er}_{2} \mathrm{O}_{3}\right)$ ensayados en agua a temperatura ambiente $\left(25^{\circ} \mathrm{C}\right)$ en función de la velocidad de deformación.
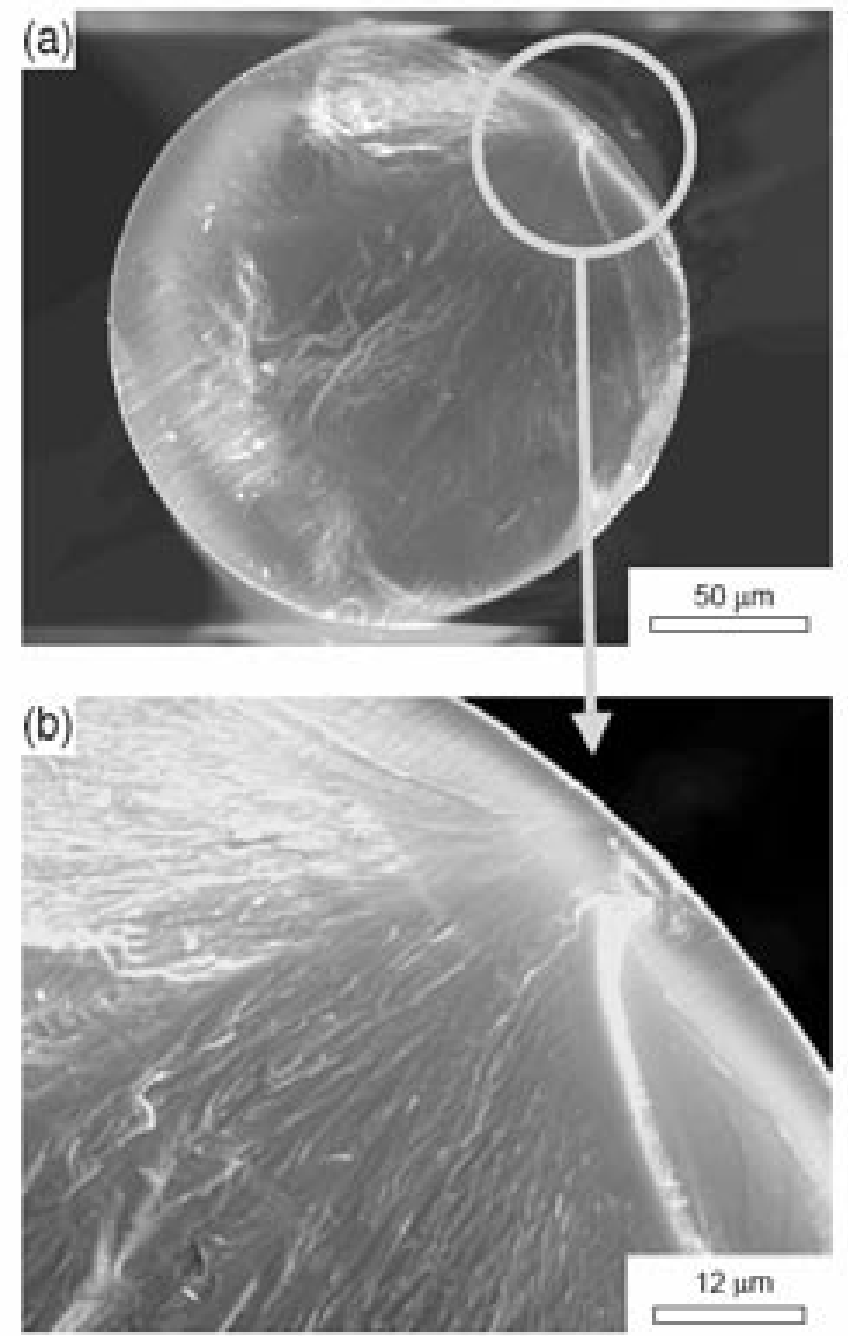

Fig. 9- Superficie de fractura de muestra ensayada a $25^{\circ} \mathrm{C}$ en agua. (a) Vista general. (b) Detalle de la zona de inicio de la fractura.

efecto sobre la resistencia. Durante los ensayos (a $25^{\circ} \mathrm{C}$ ) una gota de agua, introducida mediante una jeringa, mantenía el monofilamento permanentemente expuesto a la humedad. En la figura 8 se aprecia un ligero incremento en la resistencia media al pasar de velocidades de deformación de $1 \mu \mathrm{m} /$ min a $10 \mu \mathrm{m} / \mathrm{min}$. Al comparar con los ensayos a la misma temperatura en aire, observamos que el valor medio de la resistencia en agua a $1 \mu \mathrm{m} / \mathrm{min}(4.4 \pm 0.4 \mathrm{GPa})$ es menor que en aire (5.3 $\pm 0.6 \mathrm{GPa})$. La superficie de fractura de una de las fibras ensayadas en agua a esta velocidad se muestra en la Fig. 9. La fractura se nuclea a partir de un defecto superficial, como a $25^{\circ} \mathrm{C}$ en aire, pero su morfología, lisa, recuerda a la de las muestras ensayadas a alta temperatura.

\section{DISCUSIÓN}

Los resultados tanto de los ensayos mecánicos como del análisis fractográfico concuerdan con la hipótesis de que la resistencia de los monofilamentos de $\mathrm{ZrO}_{2}\left(\mathrm{Er}_{2} \mathrm{O}_{3}\right)$ entre 400 y $800^{\circ} \mathrm{C}$ depende directamente del crecimiento subcrítico de grietas a partir de defectos superficiales. Los resultados de los ensayos en agua apuntan en la misma dirección, puesto que 
muestran una reducción en la resistencia cuando las fibras se ensayan a baja velocidad ( $1 \mu \mathrm{m} / \mathrm{min})$. Pueden encontrarse referencias en la literatura acerca de crecimiento subcrítico de grieta (CSG) en materiales cerámicos de $\mathrm{ZrO}_{2}\left(\mathrm{Y}_{2} \mathrm{O}_{3}\right)$ después de recocidos prolongados entre 200 y $300{ }^{\circ} \mathrm{C}$ [8-9] o expuestos al agua [10-11]. Las medidas por difracción de rayos $X y$ dilatometría mostraban la formación de circona monoclínica en la superficie de las muestras, y el subsiguiente cambio de volumen asociado con la transformación producía la nucleación de grietas superficiales responsables de la pérdida de resistencia. Knechtel et al. [14] demostraron la existencia de crecimiento subcrítico de grieta a temperatura ambiente en circona estabilizada con itria ( $3 \%$ molar) cuando el factor de intensidad de tensiones en la punta de la grieta superaba determinado umbral. Chevalier et al. [15] probaron además que la velocidad de propagación de la grieta y el factor de intensidad de tensiones umbral disminuían si las muestras se sumergían en agua, mientras que el crecimiento subcrítico de grieta era prácticamente despreciable si los ensayos se realizaban en silicona líquida o en vacío.

Todos estos resultados demuestran la influencia del agua, tanto en forma líquida como de humedad ambiental sobre la $\mathrm{ZrO}_{2}\left(\mathrm{Y}_{2} \mathrm{O}_{3}\right)$ tetragonal a temperaturas intermedias. Este fenómeno siempre se había ligado a la desestabilización de la fase tetragonal, tal y como demostraba la presencia de circona monoclínica en los materiales rotos. Las teorías que trataban de explicar esta desestabilización postulaban la formación de hidróxidos ( $\mathrm{Zr}-\mathrm{OH}$ e $\mathrm{Y}-\mathrm{OH})$ por adsorción de agua en la superficie, lo que aumentaría las tensiones en la superficie y la nucleación de la transformación martensítica [10]; o bien el lixiviado del itrio por el agua, que conduciría a la desestabilización de los granos y su paso a la fase monoclínica [16]. Más recientemente, Guo [17-19] propuso un nuevo mecanismo según el cual reacciones superficiales con el ion $\mathrm{O}^{2-}$ darían lugar a iones $\mathrm{OH}^{-}$, que penetrarían en el material e irían aniquilando las vacantes de oxígeno. Si la concentración de estas vacantes cayera por debajo de determinado umbral, la fase tetragonal dejaría de ser estable y nos encontraríamos con la degradación por transformación de fase del material.

Para comprobar si alguno de estos mecanismos se daba en la $\mathrm{ZrO}_{2}\left(\mathrm{Er}_{2} \mathrm{O}_{3}\right)$, se utilizó la técnica de espectroscopia Raman confocal. La figura 10 muestra uno de los espectros representativos de las superficies de fractura (en el entorno de su lugar de inicio de la grieta) de las muestras ensayadas a $600{ }^{\circ} \mathrm{C}$ en aire. A pesar del ruido de fondo introducido por la fuerte emisión del ion $\mathrm{Er}^{3+}$, los cinco picos característicos de la circona tetragonal $\left(640,465,325,260\right.$ y $\left.150 \mathrm{~cm}^{-1}\right)$ se aprecian claramente, mientras que los 14 picos esperados para la circona monoclínica no se encontraron en ninguna de las muestras [20]. Los espectros registrados a partir del resto de muestras ensayadas mostraron los mismos resultados: no hay evidencia de circona monoclínica hasta el límite de resolución de la técnica, que en nuestro caso sería de capas menores de 40 $\mathrm{nm}$. En cualquier caso, esto supone una significativa diferencia con los casos estudiados de degradación en $\mathrm{ZrO}_{2}\left(\mathrm{Y}_{2} \mathrm{O}_{3}\right)$, donde la dicho proceso involucraba incrementos del $10 \%$ de la fase monoclínica.

El crecimiento subcrítico de grieta deberá por tanto tener un origen distinto a la degradación por transformación de fase. Existe un argumento cinético adicional a favor de esta afirmación. El crecimiento subcrítico de grieta en $\mathrm{ZrO}_{2}\left(\mathrm{Y}_{2} \mathrm{O}_{3}\right)$ aparece tras exposiciones prolongadas $(100 \mathrm{~h})$ a temperaturas entre 200 y $300{ }^{\circ} \mathrm{C}$ [8-9], mientras que la resistencia de los

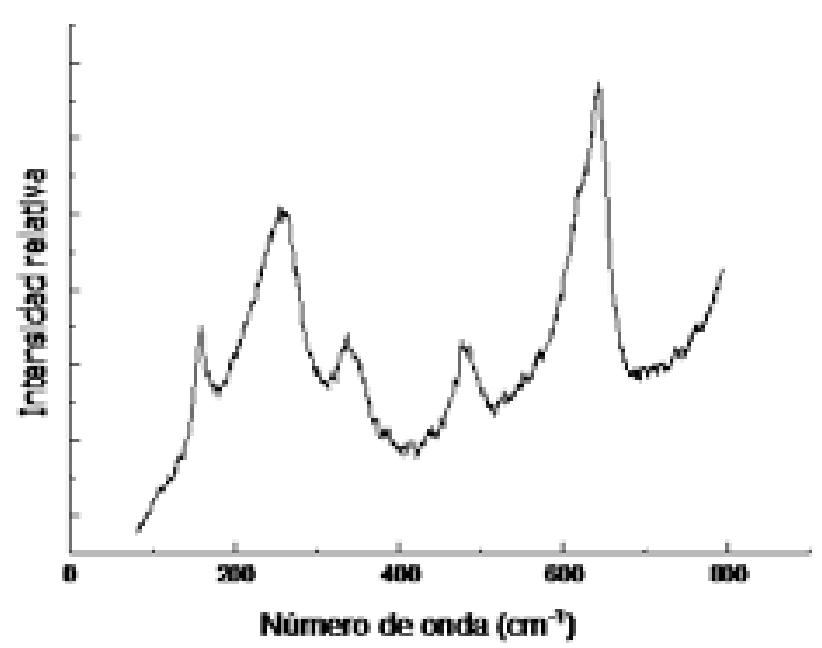

Fig. 10- Espectro Raman de la superficie de fractura (cerca del lugar de inicio de la misma) en una fibra rota a $600{ }^{\circ} \mathrm{C}$ y $1 \mu \mathrm{m} / \mathrm{min}$. Los cinco picos presentes (a 150, 260, 325, 465, y $640 \mathrm{~cm}^{-1}$ ) son los correspondientes a la estructura tetragonal.

monofilamentos de $\mathrm{ZrO}_{2}\left(\mathrm{Er}_{2} \mathrm{O}_{3}\right)$ se degradaba en ensayos que duraban de 5 a 10 minutos $(10 \mu \mathrm{m} / \mathrm{min})$ a $400{ }^{\circ} \mathrm{C}$. Entre los diferentes mecanismos capaces de activar el crecimiento subcrítico de grieta es preciso considerar la presencia de tensiones residuales generadas en la superficie de la fibra debido al fuerte gradiente térmico que se da durante la solidificación. Estas tensiones pueden sumarse a las de flexión y dar lugar a la propagación de una grieta desde cualquiera de los defectos superficiales. Para analizar su efecto, se sometió a un conjunto de fibras a un recocido a $1200{ }^{\circ} \mathrm{C}$ durante 2 horas. Puesto que el material presenta [7] deformación plástica en el sistema primario de deslizamiento $\{100\}<011>$ precisamente a $1200{ }^{\circ} \mathrm{C}$, éste tratamiento de recocido debería ser capaz de eliminar las tensiones residuales. Posteriormente, los monofilamentos recocidos se ensayaron a flexión a $800{ }^{\circ} \mathrm{C}$ y $1 \mu \mathrm{m} / \mathrm{min}$. La resistencia media a flexión resultó ser de 2.4 $\pm 0.1 \mathrm{GPa}$, prácticamente idéntico al valor de $2.4 \pm 0.2 \mathrm{GPa}$ registrado bajo las mismas condiciones en el caso no recocido. Por consiguiente, las tensiones residuales parecen no ser relevantes en la aparición de crecimiento subcrítico de grieta en $\mathrm{ZrO}_{2}\left(\mathrm{Er}_{2} \mathrm{O}_{3}\right)$.

Los resultados condensados en la figura 5 son similares a los descritos en alúmina monocristalina, que también muestra un claro deterioro de las propiedades mecánicas en el intervalo de 300 a $600{ }^{\circ} \mathrm{C}$ debido al crecimiento subcrítico de grieta [21-22]. Wiederhorn [23] descubrió que la velocidad de grieta aumentaba con la humedad relativa en monocristales de alúmina a temperatura ambiente, demostrando que éste comportamiento se debía a corrosión bajo tensión. Un mecanismo similar parece ser el responsable de la degradación con la presencia de agua y la temperatura de la $\mathrm{ZrO}_{2}\left(\mathrm{Er}_{2} \mathrm{O}_{3}\right)$ tetragonal que se presenta en el presente artículo. Esta hipótesis viene avalada por el hecho de que el crecimiento subcrítico de grieta comienza en la superficie, indicando que las condiciones ambientales influyen de manera efectiva. Newcomb y Tressler [24, 25], después de estudiar el crecimiento subcrítico de grieta en $\mathrm{Al}_{2} \mathrm{O}_{3}$ monocristalina entre 800 y $1500{ }^{\circ} \mathrm{C}$ postularon que era consecuencia de la rotura térmicamente activada de los enlaces químicos asociada con un mecanismo de atropamiento por la 
red (lattice trapping). En principio, un mecanismo de este tipo podría actuar en la $\mathrm{ZrO}_{2}\left(\mathrm{Er}_{2} \mathrm{O}_{3}\right)$ tetragonal, pero mientras que en la alúmina el crecimiento subcrítico de grietas siempre comenzaba en poros internos, en nuestro caso lo hace siempre en la superficie. Esta evidencia, junto con el efecto de la humedad a temperatura ambiente, sugiere que el crecimiento subcrítico de grieta se debe a corrosión bajo tensión, si bien no se puede descartar la contribución de otros mecanismos a alta temperatura.

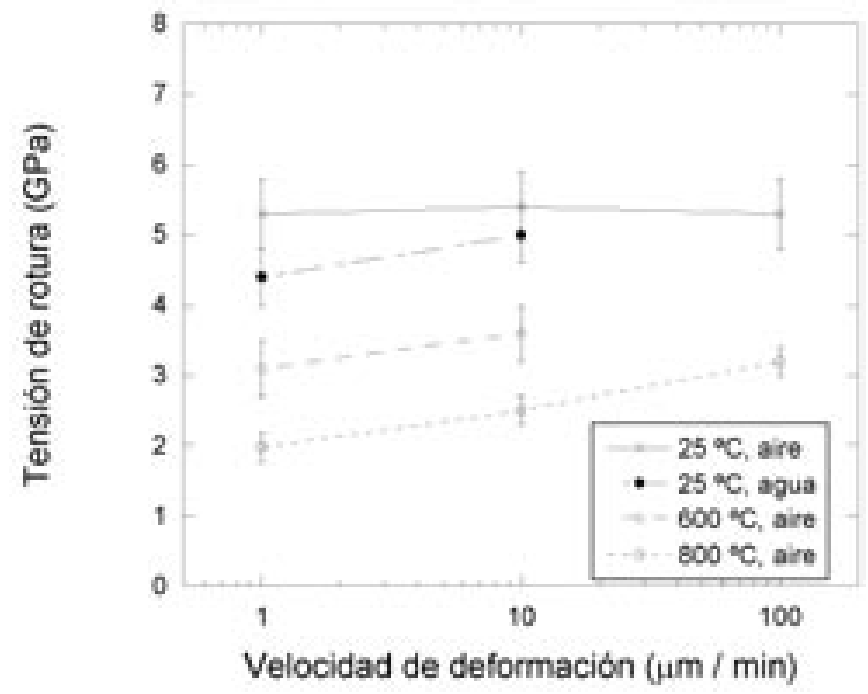

Fig. 11- Influencia de la velocidad de deformación sobre la tensión de rotura de fibras monocristalinas de $\mathrm{ZrO}_{2}\left(\mathrm{Er}_{2} \mathrm{O}_{3}\right)$ tetragonal a distintas temperaturas.

Si el crecimiento subcrítico de grieta es responsable de la dependencia con el tiempo que se observa en las Figs. 2, 3 y 4 , podemos estimar la cinética de crecimiento de la grieta en un monocristal de circona tetragonal suponiendo que la velocidad de la grieta, $v$, sigue una ley del tipo $v=A K^{n}$, donde $\mathrm{K}$ es el factor de intensidad de tensiones en la punta de la grieta, y A y n son dos constantes de dependen del material y de las condiciones ambientales. Las tensiones de rotura estarán relacionadas con las velocidades de deformación según [24-28]

$$
\sigma^{n+1}=\frac{2(n+1) E \dot{\varepsilon} \sigma_{0}^{n-2}}{A Y^{2}(n-2) K_{c}^{n-2}}
$$

donde $\mathrm{E}$ es el módulo elástico, $\dot{\varepsilon}$ es la velocidad de deformación, $\sigma_{0}$ la resistencia inerte, $K_{c}$ la tenacidad e $Y$ una constante geométrica. A partir de la pendiente de la gráfica $\log \sigma-\log \dot{\varepsilon}$ se puede obtener $n$. Los resultados se muestran en la Tabla 1. Las magnitudes de $\mathrm{n}$ a 600 y $800{ }^{\circ} \mathrm{C}$ son similares a los de la alúmina [23], donde la propagación de la grieta se debía a corrosión bajo tensión. Por otro lado, los valores de $\mathrm{n}$ a temperatura ambiente en agua y a $600{ }^{\circ} \mathrm{C}$ en aire son

TABLA 1. ESTIMACIÓN DEL EXPONENTE N DE CRECIMIENTO DE GRIETA EN FUNCIÓN DE LA TEMPERATURA Y DEL MEDIO. VALORES DE N GRANDES INDICAN MENOR SUSCEPTIBILIDAD A CORROSIÓN BAJO TENSIÓN.

\begin{tabular}{|c|c|c|c|c|}
\hline Temperatura $\left({ }^{\circ} \mathrm{C}\right)$ & $25^{\circ} \mathrm{C}$ & $25^{\circ} \mathrm{C}$ & $600{ }^{\circ} \mathrm{C}$ & $800{ }^{\circ} \mathrm{C}$ \\
\hline Ambiente & Aire & Agua & Aire & Aire \\
\hline $\mathrm{n}$ & $>200$ & 16.3 & 16.9 & 8.8 \\
\hline
\end{tabular}

similares, lo que indica que tenemos un fenómeno que se puede activar bien por la temperatura o bien aumentando la humedad ambiente.

\section{CONCLUSIONES}

Se ha estudiado la susceptibilidad ambiental de las fibras monocristalinas de $\mathrm{ZrO}_{2}\left(\mathrm{Er}_{2} \mathrm{O}_{3}\right)$ tetragonal fabricados mediante fusión zonal por láser mediante ensayos de flexión en tres puntos en aire entre 25 y $800{ }^{\circ} \mathrm{C}$ y en agua a temperatura ambiente. La resistencia media a flexión decae acusadamente con la temperatura y es menor en agua que en aire. Del mismo modo, la resistencia a flexión de los monofilamentos ensayados en las condiciones anteriormente mencionadas aumenta con la velocidad de deformación. Este comportamiento se refleja en cambios en la morfología de las superficies de fractura: mientras que las fibras ensayadas a temperatura ambiente muestran superficies rugosas y se encuentra que la fractura se nuclea en pequeños defectos superficiales, las ensayadas a alta temperatura son más lisas y cuentan con una zona especular (mirror) de apreciable extensión consecuencia del crecimiento subcrítico de grieta en torno a un defecto superficial.

Los mecanismos responsables del crecimiento subcrítico de grieta en $\mathrm{ZrO}_{2}\left(\mathrm{Er}_{2} \mathrm{O}_{3}\right)$ difieren de los observados en $\mathrm{ZrO}_{2}\left(\mathrm{Y}_{2} \mathrm{O}_{3}\right)$ tetragonal. En particular, no ha aparecido fase monoclínica en ninguna de las muestras, aun en las que experimentaban mayor degradación de sus propiedades mecánicas, mientras que la aparición de fase monoclínica siempre se presenta en las superficies de fractura de $\mathrm{ZrO}_{2}\left(\mathrm{Y}_{2} \mathrm{O}_{3}\right)$ con algún grado de susceptibilidad ambiental. El recocido efectuado para aliviar las tensiones residuales provenientes del procesado no altera el comportamiento mecánico de los monofilamentos, por lo que se puede extraer la conclusión de que el crecimiento subcrítico de grieta en $\mathrm{ZrO}_{2}\left(\mathrm{Er}_{2} \mathrm{O}_{3}\right)$ monocristalina se debe a corrosión bajo tensión, de forma similar a la referida en alúmina monocristalina.

\section{AGRADECIMIENTOS}

Este trabajo ha sido financiado por el Ministerio de Educación y Ciencia a través del proyecto de investigación MAT2003-6085, MAT2006-13005-C03-02 y por la Comunidad de Madrid a través del proyecto GR/MAT/0357/2004, por lo cual queremos expresar nuestro más sincero agradecimiento.

\section{BIBLIOGRAFÍA}

1. R. C. Garvie, R. R. Hughan, and R. T. Pascoe, "Ceramic steel," Nature (London), 258 703-704 (1973).

2. R. H. J. Hannink and M. V. Swain, "Progress in transformation toughening of ceramics," Ann. Rev. Mater. Sci., 24 359-408 (1994).

3. R. H. J. Hannink, P. M. Kelly, and B. C. Muddle, "Transformation toughening in zirconia-containing Ceramics ," J. Am. Ceram. Soc., 83 [3] 461-87 (2000).

4. P. Duran, "The system erbia-zirconia", J. Am. Ceram. Soc., 60 [11-12] 510-3 (1977).

5. M. Yashima, N. Ishizawa, T. Noma, and M. Yoshimura, “Stable and metastable phase relationships in the system $\mathrm{Er}_{2} \mathrm{O}_{3}-\mathrm{ZrO}_{2}$ '" J. Am. Ceram. Soc., 74 [3] 510-3 (1991).

6. M. Yashima, T. Noma, N. Ishizawa, and M. Yoshimura, "Effect of noncompositional inhomogeneity on $\mathrm{t}$ à $\mathrm{m}$ phase transformation during grinding of various rare-earthdoped zirconias," J. Am. Ceram. Soc., 74 [12] 3011-6 (1991).

7. J. Martinez-Fernández, A.R. Pinto Gómez, J.J. Quispe-Cancap, A.R. de Arellano-López J. Llorca, J.Y. Pastor, S. Farmer, A. Sayir, “High-temperature plastic deformation of $\mathrm{Er}_{2} \mathrm{O}_{3}$-doped $\mathrm{ZrO}_{2}$ single crystals", Acta Materialia, 54, 2195-2204 (2006). 
8. M. Matsui, S. Soma, and I. Oda, "Strength of Y-TZP components"; pp. 37190 in Advances in Ceramics, vol. 12, Science and Technology of Zirconia II. Edited by N. Claussen, M. Rühle and A. H. Heuer. American Ceramic Society, Westerville, Ohio, 1984.

9. M. Watanabe, S. Iio, and I. Fukuura "Aging Behavior of Y-TZP"; pp. 391-8 in Advances in Ceramics, vol. 12, Science and Technology of Zirconia II. Edited by N. Claussen, M. Rühle and A. H. Heuer. American Ceramic Society, Westerville, Ohio, 1984.

10. M. Yoshimura, T. Noma, K. Kawabata, and S. Somiya, "Role of $\mathrm{H}_{2} \mathrm{O}$ on the degradation processes of Y-TZP," J. Mater. Sci. Lett., 6 465-7 (1987).

11. T. Sato and M. Shimada, "Transformation of yttria-doped tetragonal $\mathrm{ZrO}$ polycrystals by annealing in water," J. Am. Ceram. Soc. 68 [6] 356-9 (1985).

12. A. Sayir, S. C. Farmer, P. O. Dickerson and H. M. Yun, "High temperature mechanical properties of $\mathrm{Al}_{2} \mathrm{O}_{3} / \mathrm{ZrO}_{2}\left(\mathrm{Y}_{2} \mathrm{O}_{3}\right)$ fibres," Mat. Res. Soc. Symp. Proc. 365, 21-7 (1995).

13. A. Sayir, and S. C. Farmer, "The effect of microstructure on mechanical properties of directionally solidified $\mathrm{Al}_{2} \mathrm{O}_{3}-\mathrm{ZrO}_{2}\left(\mathrm{Y}_{2} \mathrm{O}_{3}\right)$ eutectic," Acta mater., 48 4691-7 (2000).

14. M. C. Knechtel, D. E. Garcia, J. Rödel and N. Claussen, "Subcritical crack growth in Y-TZP and Al2O3-toughened Y-TZP," J. Am. Ceram. Soc., 76 [10] 2681-4 (1993).

15. J. Chevalier, C. Olagnon, G. Fantozzi, "Subcritical crack growth in 3Y-TZP ceramics: static and cyclic fatigue," J. Am. Ceram. Soc., 82 [11] 3129-38 (1999).

16. F. F. Lange, G. L. Dunlop and B. I. Davis, "Degradation during aging of transformation-toughened of $\mathrm{ZrO}_{2}-\mathrm{Y}_{2} \mathrm{O}_{3}$ materials at $250^{\circ} \mathrm{C}$," J. Am. Ceram. Soc., 69 (3)237-40 (1986).

17. X. Guo, "Hydrothermal degradation mechanism of tetragonal zirconia," J. Mater. Sci., 36 3737-43 (2001).
18. X. Guo and J. He, "Hydrothermal degradation of cubic zirconia," Acta mater. 51 5123-30 (2003)

19. X. Guo, "Property degradation of tetragonal zirconia induced by lowtemperature defect reaction with water molecules, "Chem. Mater., 16 [21] 3988-94 (2004).

20. A. Feinberg and C. H. Perry, "Structural Disorder and Phase Transitions in $\mathrm{ZrO}_{2}-\mathrm{Y}_{2} \mathrm{O}_{3}$ System," J. Phys. Chem. Solids, 42, 513-8 (1981), and references therein.

21. J. B. Watchman and L. H. Maxwell, "Strength of synthetic single crystal sapphire and ruby as a function of temperature," J. Am. Ceram. Soc., 42 432-3 (1959).

22. H. Sayir, A. Sayir, and P. Lagerlof, "Temperature-dependent brittle fracture of undoped and impurity doped saphhire fibers," Ceram. Engng. Sci. Proc., 14 581-90 (1993).

23. S. M. Wiederhorn, "Moisture assisted crack growth in ceramics," Int. J. Frac. Mech., 4 [2] 171-7 (1968).

24. S. A. Newcomb and R. E. Tressler, "Slow crack growth in sapphire fibers at $800^{\circ} \mathrm{C}$ to $1500^{\circ} \mathrm{C}^{\prime \prime}$ J. Am. Ceram. Soc., 76 [10] 2505-12 (1993).

25. J.J. Quispe-Cancapa, A.R. de Arellano-López, J. Martínez-Fernández, A. Sayir, "Tensile strength of directionally solidified chromia-doped sapphire", J. Eur. Ceram. Soc., 25, 1259-68 (2005).

26. A. Ridruejo, J.Y. Pastor, P. Poza, J. LLorca, A. Sayir, y V.M. Orera, “Stress corrosion cracking of single-crystal tetragonal $\mathrm{Er}_{2} \mathrm{O}_{3}$-doped $\mathrm{ZrO}_{2}{ }_{2}$, J. $\mathrm{Am}$. Ceram. Soc., 88 [11] 3125-30 (2005).

27. J.Y. Pastor, J. LLorca, P. Poza, J.J. Quispe, A.R. de Arellano-López, J. Martínez-Fernández, A. Sayir, y V.M. Orera, "High-temperature tensile strength of $\mathrm{Er}_{2} \mathrm{O}_{3}$-doped $\mathrm{ZrO}_{2}$ single crystals", J. Am. Ceram. Soc., 89 [7] 2140-46 (2006).

28. A. G. Evans, "Slow crack growth in brittle materials under dynamic loading conditions," Int. J. Fract. Mech., 10 [2] 251-9 (1974).

Recibido: $\quad 07.06 .05$

Aceptado: 13.12 .06 